\title{
Life Style-Related Diseases of the Digestive System: Colorectal Cancer as a Life Style-Related Disease: from Carcinogenesis to Medical Treatment
}

\author{
Hirokazu Takahashi ${ }^{1}$, Kyoko Yoneda ${ }^{1}$, Ayako Tomimoto ${ }^{1}$, Hiroki Endo ${ }^{1}$, Toshio Fujisawa ${ }^{1}$, Hiroshi Iida ${ }^{1}$, \\ Hironori Mawatari ${ }^{1}$, Yuichi Nozaki ${ }^{1}$, Tamon Ikeda ${ }^{1}$, Tomoyuki Akiyama ${ }^{1}$, Masato Yoneda ${ }^{1}$, \\ Masahiko Inamori ${ }^{1}$, Yasunobu Abe ${ }^{1}$, Satoru Saito ${ }^{1}$, Atsushi Nakajima ${ }^{1, *}$, and Hitoshi Nakagama ${ }^{2}$ \\ ${ }^{I}$ Gastroenterology Division, Yokohama City University Graduate School of Medicine, \\ 3-9 Fuku-ura, Kanazawa-ku, Yokohama 236-0004, Japan \\ ${ }^{2}$ Biochemistry Division, National Cancer Center Research Institute, Tokyo 104-0045, Japan
}

Received July 10, 2007; Accepted September 10, 2007

\begin{abstract}
Life style-related diseases are associated with an increased risk of colorectal cancer (CRC). Recently, an association has been demonstrated between obesity and CRC. CRC has been associated with markers of insulin or glucose control, and insulin resistance might be the unifying mechanism by which several risk factors affect colorectal carcinogenesis. We evaluated the association between the number of aberrant crypt foci (ACF) and obesity, insulin resistance, hyperlipidemia, and other factors of life style-related disease. As a result, age, body mass index (BMI), waist circumference, and visceral fat obesity were significantly associated with the number of ACF. These results suggest that visceral fat obesity is an important target for CRC prevention. Peroxisome proliferator-activated receptor gamma $(\operatorname{PPAR} \gamma)$ is a member of the nuclear receptor superfamily and is highly expressed in CRC. PPAR $\gamma$ ligand administration for 1 to 8 months significantly reduced the number of ACF in human subjects. PPAR $\gamma$ ligand is a promising candidate as a chemopreventive agent. Further investigation is needed to elucidate these mechanisms.
\end{abstract}

Keywords: life style-related disease, colorectal cancer, chemoprevention, aberrant crypt foci (ACF), peroxisome proliferator-activated receptor gamma (PPAR $\gamma$ )

\section{Introduction}

A diet rich in fat and calories and low intake of vegetable, fruits, and fibers are referred to as a Western diet. Chronic conditions including obesity, diabetes, hyperlipedemia, hypertension, and cardiovascular disease have been shown to be associated with a Western diet, alcohol intake, and smoking. Indeed, obesity has been reported to be associated with an elevated risk of cardiovascular disease, diabetes, and mortality $(1-4)$. Especially, visceral fat obesity is increasing and is becoming a significant social problem. Recently, these life styles have also been shown to be correlated with

*Corresponding author. nakajima-tky@umin.ac.jp

Published online in J-STAGE: October 6, 2007

doi: 10.1254 /jphs.FM0070022 increase in colorectal cancer (CRC) risk.

$\mathrm{CRC}$ is a disease with a high mortality and morbidity rate, and currently, its prevalence has been increasing worldwide. On the other hand, CRC is potentially one of the most preventable malignancies $(5,6)$. Correction of the life styles mentioned above may have a major potential for CRC prevention. On the other hand, early detection of CRC or CRC precursor lesions may be promoted by screening of the population at high risk. In addition, chemoprevention, the use of medications to prevent disease, has now been extensively explored in CRC. Some of these interventions, such as supplemental fibers, calcium supplementation, aspirin, nonsteroidal anti-inflammatory drugs (NSAIDs), and selective cyclooxygenase (COX)-2 inhibitors, have been shown to have a potential to reduce both CRC and colorectal adenomas. $\mathrm{CRC}$ is thought to progress through several morpho- 
logical stages, from the formation of adenomatous polyps to malignant conversion (7). Genetic alterations including mutations in the $A P C, K$-ras, and $p 53$ genes have been reported to accompany the disease progression (8). The earliest identifiable lesion in this pathway may be the aberrant crypt foci (ACF). ACF are prepolyp abnormalities identified in single crypts by magnifying colonoscopy after the administration of methlyene blue dye. Therefore, ACF may be a surrogate marker of $\mathrm{CRC}$, and analysis of the association between clinicopathological variables and ACF formation may be of great significance.

\section{Diabetes, obesity, and CRC}

Diabetes, obesity, hyperinsulinemia, and insulin resistance have been repeatedly shown to be associated with CRC (9-14). Diabetes was associated with an increased risk for CRC in cohort studies, in accordance with previous studies that demonstrated that diabetes is a moderate risk factor for CRC. Overweight, obesity, or high BMI has been consistently associated with increased risk for CRC incidence and mortality, at least in men and premenopausal women $(15-18)$. The WHO definition of the life style-related disease allows the use of a body mass index (BMI) of at least $30 \mathrm{~kg} / \mathrm{m}^{2}$ instead of waist circumference or waist-to-hip ratio (19).

\section{ACF as a biomarker of CRC}

ACF, which represent clusters of aberrant colorectal crypts, were first discovered in mice treated with azoxymethane (20). ACF have been demonstrated to be precursor lesions of $\mathrm{CRC}$, and with further investigations, ACF have been established as a biomarker of the risk of CRC in azoxymethane-treated mice and rats (21). In humans, the relationship between ACF and CRC is less clear. The number of ACF was measured using magnifying endoscopy, but the association between ACF and CRC was only partially evident. ACF, however, are also thought to be precursor lesions of colorectal adenoma and CRC in humans (22). Many factors are known to be associated with increased or decreased risk of CRC. Among them, only history of adenomas, age, and the use of NSAIDs have been examined in relation to the development of ACF. The relationships of other factors to the occurrence of $\mathrm{ACF}$ in the colon remain unknown.

In the rat model, the formation of ACF was enhanced by cancer promoters and suppressed by chemopreventive agents (23). ACF has also been reported in colonic mucosa in humans $(24,25)$. Patients with CRC had more ACF with $K$-ras mutations than those without
Table 1. Relationships between selected risk factors for colorectal cancer and the number of ACF in the colorectum

\begin{tabular}{llc}
\hline Risk factors & Correlation coefficient & $P$-value \\
\hline Age (years) & 0.256 & $0.0121^{*}$ \\
BMI & 0.263 & $0.0044^{*}$ \\
Waist (cm) & 0.370 & $0.0003^{*}$ \\
Fasting blood sugar & 0.021 & 0.7575 \\
HOMA-IR & 0.263 & 0.6174 \\
Total cholesterol & 0.263 & 0.4771 \\
Triglyceride & 0.263 & 0.2049 \\
VFA $\left(\mathrm{cm}^{2}\right)$ & 0.512 & $<0.0001^{*}$ \\
SFA $\left(\mathrm{cm}^{2}\right)$ & 0.108 & 0.2091 \\
\hline
\end{tabular}

$* P$-values $<0.05$ were considered to denote statistical significance. HOMA-IR: homeostasis model assessment of insulin resistance.

CRC. These results suggest that ACF are not only morphologically but also genetically distinct lesions and are precursors of adenoma and CRC.

\section{ACF and visceral fat obesity}

The association between the number of ACF and age, BMI, waist circumference, diabetes, serum lipid, visceral fat area (VFA), and subcutaneous fat area (SFA) were evaluated (Table 1). Our findings indicate that age, BMI, waist circumference, and VFA were significantly associated with the number of ACF. Especially, VFA was strongly associated with the number of ACF. Visceral fat tissue is known as an endocrine organ that secretes adipocytokines such as TNF- $\alpha$, leptin, and adiponectin. These adipocytokines and/or the visceral fat itself may play an important role in colon carcinogenesis. The number of ACF increased with age. Genetic and epigenetic alterations accumulate with advancing of age; therefore, the increased risk of $\mathrm{ACF}$ formation with age may be mainly influenced by these genetic alterations.

\section{Chemoprevention for CRC}

Chemoprevention, the use of medications to prevent disease, has now been extensively explored in CRC. Supplemental fibers, calcium supplementation, aspirin, NSAIDs, and selective COX-2 inhibitors have a potential to reduce both CRC and colorectal adenomas. Higher doses and longer durations of use of NSAIDs and COX-2 inhibitors seem to be associated with greater protection from CRC and adenoma. However, these agents are associated with significant cardiovascular events and/or gastrointestinal damage. The balance of benefits to risk does not favor chemoprevention by these 


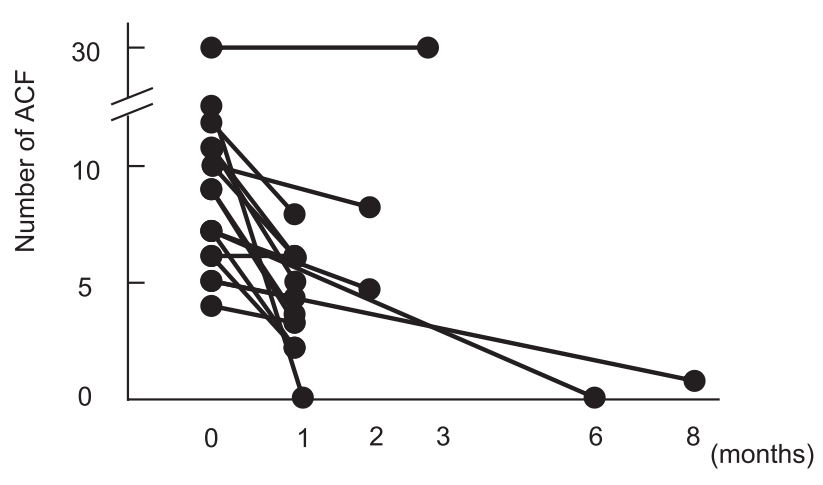

Fig. 1. The numbers of ACF were prospectively examined in 14 subjects before and after $1-8$ months of pioglitazone treatment. After PPAR $\gamma$ ligand treatment, the number of ACF significantly decreased.

agents in average-risk individuals.

\section{ACF and PPAR $\gamma$}

$\operatorname{PPAR} \gamma$ is mainly expressed in adipose tissue and plays a central role in adipocyte differentiation and insulin sensitivity. Activating synthetic ligands for $\operatorname{PPAR} \gamma$, such as pioglitazone, are commonly used to treat diabetes. PPAR $\gamma$ is also overexpressed in many tumors. This suggests that modulation of PPAR $\gamma$ expression or function might have impact on tumor cell survival. Chemopreventive effects of PPAR $\gamma$ ligand on the formation of the human ACF were evaluated. Fourteen patients were examined for ACF by magnifying colonoscopy before and after 1 to 8 months of pioglitazone treatment (Fig. 1). After PPAR $\gamma$ ligand treatment, the number of ACF was significantly decreased. These results suggest that a PPAR $\gamma$ ligand is a promising candidate as a chemopreventive agent for CRC.

\section{Conclusions}

Our results suggest that visceral fat obesity may be a risk factor for CRC and visceral fat may play an important role in colorectal carcinogenesis at an earlier stage in the adenoma-carcinoma sequence (25). Adipocytokines secreted by visceral fat tissue and/or the visceral fat itself may play an important role in colon carcinogenesis. PPAR $\gamma$ ligand is a promising candidate for $\mathrm{CRC}$ chemoprevention.

\section{Acknowledgments}

We thank Masako Ochiai and Machiko Hiraga for their technical assistance. This work was supported in part by a Grant-in-Aid for research on the Third Term
Comprehensive Control Research for Cancer from the Ministry of Hearth, Labour, and Welfare, Japan to A.N.; a grant from the National Institute of Biomedical Innovation (NBIO) to A.N.; a grant from the Ministry of Education, Culture, Sports, Science, and Technology, Japan (KIBAN-B) to A.N.; a grant from the Ministry of Education, Culture, Sports, Science, and Technology, Japan (WAKATE-B) to H.T.; a research grant of the Princess Takamatsu Cancer Research Fund to A.N.; and a grant for 2007 Strategic Research Project (No. K19041) of Yokohama City University, Japan to H.T. and A.N.

\section{References}

1 Fujioka S, Matsuzawa Y, Tokunaga K, Tarui S. Contribution of intra-abdominal fat accumulation to the impairment of glucose and lipid metabolism in human obesity. Metabolism. 1987;36: 54-59.

2 Larsson B, Svardsudd K, Welin L, Wilhelmsen L, Björntorp P, Tibblin G. Abdominal adipose tissue distribution, obesity, and risk of cardiovascular disease and death: 13 year follow up of participants in the study of men born in 1913. Br Med J. 1984;288:1401-1404.

3 Kissebah AH, Vydelingum N, Murray R, Evans DJ, Hartz AJ, Kalkhoff RK, et al. Relation of body fat distribution to metabolic complications of obesity. J Clin Endocrinol Metab. 1982;54: 254-260.

4 Folsom AR, Kaye SA, Sellers TA, Hong CP, Cerhan JR, Potter $\mathrm{JD}$, et al. Body fat distribution and 5-year risk of death in older women. JAMA. 1993;269:483-487.

5 Anderson WF, Umar A, Brawley OW. Colorectal carcinoma in black and white race. Cancer Metastasis Rev. 2003;22:67-82.

6 Rougier P, Mitry E. Epidemiology, treatment and chemoprevention in colorectal cancer. Ann Oncol. 2003;14:ii3-ii5.

7 Kinzler KW, Vogelstein B, Lessons from hereditary colorectal cancer. Cell. 1996;87:159-170.

8 Fearon ER, Vogelstein B. A genetic model for colorectal tumorigenesis. Cell. 1990;61:759-767.

9 Limburg PJ, Anderson KE, Johnson TW, Jacobs DR, Lazovich $\mathrm{D}$, Hong $\mathrm{CP}$, et al. Diabetes mellitus and subsite-specific colorectal cancer risks in the Iowa Women's Health Study. Cancer Epidemiol Biomarkers Prev. 2005;14:133-137.

10 Chang CK, Ulrich CM. Hyperinsulinaemia and hyperglycaemia: possible risk factors of colorectal cancer among diabetic patients. Diabetologia. 2003;46:595-607.

11 Larsson SC, Giovannucci E, Wolk A. Diabetes and colorectal cancer incidence in the cohort of Swedish men. Diabetes Care. 2005;28:1805-1809.

12 Hu FB, Manson JE, Liu S, Hunter D, Colditz GA, Michels KB, et al. Prospective study of adult onset diabetes mellitus (type 2) and risk of colorectal cancer in women. J Natl Cancer Inst. 1999;91:542-547.

13 Schoen RE, Tangen CM, Kuller LH, Burke GL, Cushman M, Tracy RP, et al. Increased blood glucose and insulin, body size, and indent colorectal cancer. J Natl Cancer Inst. 1999;91:11471154 .

14 Giovannucci E, Aasherio A, Rimm EB, Colditz GA, Stampfer 
MJ, Willett WC. Physical activity, obesity, and risk for colon cancer and adenoma in men. Ann Intern Med. 1995;122:327334.

15 Frezza EE, Wachtel MS, Chiriva-Internati M. Influence of obesity on the risk of developing colon cancer. Gut. 2006;55: 285-291.

16 Slattery ML, Ballard-Barbash R, Edwards S, Caan BJ, Potter JD. Body mass index and colon cancer: an evaluation of the modifying effects of estrogen (United States). Cancer Causes Control. 2003; $14: 75-84$.

17 Giovannucci E. Diet, body weight, and colorectal cancer: a summary of the epidemiologic evidence. J Womens Health. 2003;12:173-182.

18 El-Serag HB. Obesity and disease of the oesophagus and colon. Gastroenterol Clin North Am. 2005;34:63-82.

19 World Health Organization. Definition, diagnosis, and classification of diabetes mellitus and its complications: report of a WHO consultation. Geneva: World Health Organization; 1999.

20 Bird RP. Observation and quantification of aberrant crypts in the murine colon treated with a colon carcinogen: preliminary findings. Cancer Lett. 1987;37:147-151.

21 Pretlow TP, O’Riordan MA, Somich GA, Amini SB, Pretlow TG. Aberrant crypts correlate with tumor incidence in F344 rats treated with azoxymethane and phytate. Carcinogenesis. 1992;13:1509-1512.

22 Takayama T, Katsuki S, Takahashi Y, Ohi M, Nojiri S, Sakamaki S, et al. Aberrant crypt foci of the colon as precursors of adenoma and cancer. N Engl J Med. 1998;339:1277-1284.

23 Sutherland LA, Bird RP. The effect of chenodeoxycholic acid on the development of aberrant crypt foci in the rat colon. Cancer Lett. 1994;76:101-107.

24 Roncucci L, Stamp D, Medline A, Cullen JB, Bruce WR. Identification and quantification of aberrant crypt foci and microadenomas in the human colon. Hum Pathol. 1991;22:287294.

25 Pretlow TP, Barrow BJ, Ashton WS, O'Riordan MA, Pretlow TG, Jurcisek JA, et al. Aberrant crypts: putative preneoplastic foci in human colonic mucosa. Cancer Res. 1991;51:1564-1567. 